\title{
Problèmes hydrauliques posés par les centrales ETM à cycle direct au large
}

\author{
Hydraulic problems posed by direct-cycle TES stations offshore
}

\author{
M.F. Gauthier et J. Zaoui \\ Sogreah, Grenoble
}

\section{Introduction}

En 1979, le groupement CGE-Alsthom Atlantique entreprenait à l'initiative du CNEXO et avec sa collaboration une étude paramétrique de faisabilité d'une centrale ETM dans la gamme de puissance électrique nette de 1 à $10 \mathrm{MW}$.

Cette étude a mis en évidence l'intêrêt économique de la solution centrale flottante en cycle direct.

En effet, pour une centrale à terre, outre que les sites potentiels sont limités, le tuyau d'amenée d'eau froide pose des problèmes techniques très difficiles et grève le bilan économique global. Cela explique la préférence donnée à une solution flottante, moins coûteuse et moins risquée.

Les solutions à cycle direct, c'est-à-dire dans lesquelles la vapeur turbinée est formée par vaporisation partielle de l'eau de la source chaude sont intéressantes à plusieurs titres notamment sur le plan économique.

Par contre, cette solution flottante à cycle direct pose toute une série de problèmes hydrauliques que nous allons passer en revue.

Dans une première partie, nous définirons les circuits, leurs interactions et la décomposition en problèmes partiels.

Une seconde partie sera consacrée aux problèmes d'hydrodynamique externe.

La dernière partie traitera de problèmes d'hydrodynamique interne.

\section{Système étudié}

La solution nominale de base utilise :

$-15 \mathrm{~m}^{3} / \mathrm{s}$ à $27^{\circ}$ comme source chaude et rejetés à $24^{\circ}$

$-8 \mathrm{~m}^{3} / \mathrm{s}$ à $4^{\circ}$ comme source froide puisés à la profondeur de $1000 \mathrm{~m}$ et rejetés à $9^{\circ}$.
Le rendement thermodynamique du cycle est de $4,4 \%$ et le rendement net de l'installation de $3 \%$ environ. Il en résulte l'impérieuse nécessité d'éviter toute dépense énergétique inutile (perte de charge, auxiliaires).

De ce fait, l'évaporateur retenu fonctionne en colonne barométrique à pression absolue constante.

L'ensemble des organes électromécaniques (évaporateurs, condenseurs, alternateurs et auxiliaires), d'une masse en ordre de marche de $5000 \mathrm{~T}$, sont formés de deux unités identiques placées sur une barge de $3500 \mathrm{~T}$ lège.

Cette dernière est soumise aux solicitations de la mer. Le tuyau d'eau froide d'un diamètre de $3,5 \mathrm{~m}$ présente une inertie totale de $10000 \mathrm{~T}$. Il doit donc être découplé mécaniquement et hydrauliquement de la barge.

La disposition générale retenue est représentée sur la figure ci-contre.

L'eau chaude prélevée sous la coque du navire, respectivement à l'avant et à l'arrière de la barge pour chacune des unités, est amenée dans un évaporateur $(E)$. La fraction non évaporée est restituée tandis que la vapeur actionne un groupe turbo-alternateur $(T A)$ avant de passer dans le condenseur $(C)$, du type à mélange ou à surface.

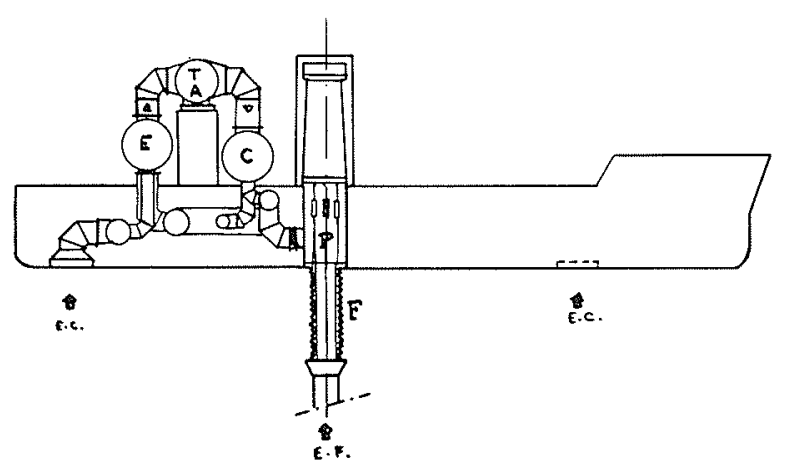

Figure 1

LA HOUILLE BLANCHE/N $/ N^{\circ} 4 / 5-1981$ 
L'alimentation en eau froide nécessaire à ce dernier s'effectue à partir d'un puit $(P)$ à surface libre aménagé au centre de la barge. Le tuyau d'arrivée d'eau froide est relié à ce puits par un raccord flexible $(F)$ étanche. Le poids apparent du tuyau d'eau froide est repris par un dispositif adéquat pour découpler ce tuyau et la barge (système antipilonnement seatankco à flotteur ou $A C B$ à vérins et accus oléopneumatiques).

Les rejets sont situés au voisinage du puits $P$. Nous verrons au paragraphe suivant les dispositions prises pour éviter à la fois les risques de recirculation et la présence d'un tuyau de rejet de grande dimension. Il en est résulté la localisation des prises d'eau chaude à l'avant et à l'arrière.

Les principaux problèmes examinés sont traités dans l'ordre suivant :

- hydraulique externe :

- rejets d'eau,

- aspirations d'eau ;

- le mouvement de la plate-forme ;

- hydraulique interne.

\section{Hydraulique externe}

\section{Rejets d'eau}

Les travaux de Craya pour le compte de la Société pour l'Energie Thermique des Mers permettent facilement d'établir qu'il n'y a aucun risque de recirculation à la source froide. Par contre, il existe un risque majeur de recirculation à la source chaude : l'écart thermique entre aspiration et rejet n'est en effet que de $3^{\circ}$. Même à vitesse d'éjection faible, le rejet se diluera dans les eaux ambiantes, créant des masses d'eau à des températures inférieures de quelques dixièmes de degré seulement à celle de l'aspiration. Vu les masses d'eau mise en jeu $\left(1,310^{6} \mathrm{~m}^{3} /\right.$ jour $)$, une recirculation est inévitable. Compter sur un courant permanent perpendiculairement à la barge constituerait une vue de l'esprit.

La solution triviale à ce problème consiste à mélanger ce rejet à des eaux plus froides soit en les réinjectant en profondeur, soit beaucoup plus simplement en mélangeant préalablement rejet chaud et rejet froid. On obtient alors une eau de température $18,8^{\circ}$.

On a primitivement pensé qu'il était nécessaire de réinjecter ce mélange au niveau de la thermocline permanente pour éviter tout risque, par exemple à $100 \mathrm{~m}$ de profondeur.

Mais il est rapidement apparu que cette disposition serait superflue car un tel mélange, nettement plus dense que les eaux de surface, plonge naturellement sur une profondeur d'au moins 50 mètres.

Le seul risque réel de recirculation apparaît dans le cas où la barge est orientée dans le sens d'un courant permanent. Mais alors le renouvellement des eaux chaudes étant assuré par le courant, il suffit de dispositions simples pour éviter une recirculation sensible (modalités de rejet et distance entre prise et rejet).

\section{Prises d'eau}

La localisation de la prise d'eau froide est aisée. Ainsi que nous l'avons évoqué au paragraphe précédent, les résultats obtenus par Craya au Laboratoire Dauphinois d'Hydraulique en 1946 sont applicables et montrent que, malgré le très faible gradient de stratification existant en profondeur, la zone verticale d'influence de la prise d'eau froide se limite à une centaine de mètres. Il n'y a donc aucune précaution particulière, hormis de rester à une distance suffisante du fond pour éliminer le risque de salissures biologiques.

La prise d'eau chaude nécessite par contre des précautions : l'extraction des gaz incondensables peut être très pénalisante. On doit donc impérativement éviter les eaux très superficielles qui pourraient entraîner une quantité notable de gaz par mer agitée.

Par ailleurs le circuit d'aspiration d'eau chaude débouche à pression constante dans le condenseur. On doit donc également le soustraire dans la mesure du possible à l'influence de la houle. Enfin l'implantation doit éviter tout risque de recirculation avec le rejet tiède.

L'étude de ce problème a montré que ces conditions sont compatibles et conduisent à situer ces prises à une trentaine de mètres de part et d'autre des rejets dans la partie basse de la barge. Les dispositions exactes devront naturellement être étudiées dans le cadre d'un projet concret.

\section{Etat de la mer}

L'incidence de l'état de la mer doit être considérée bien entendu au plan de la survie mais aussi pour déterminer le risque d'indisponibilité de la centrale. Nous nous limiterons dans cet exposé à ce second aspect.

La première difficulté provient en général de l'absence de mesures réellement fiables et de durée suffisante. Pour le secteur de Tahiti, zone potentielle possible, on dispose de deux sources distinctes, Météorologie Nationale d'une part (1484 observations en dix ans) et NOAA $^{(1)}$ d'autre part (4541 observations sur 25 ans). Ces deux sources sont en concordance satisfaisante, malheureusement cela ne suffit pas car l'une et l'autre sont basées sur des estimations visuelles dont la précision reste à établir.

La houle étant caractérisée par une période et une amplitude caractéristiques, il convient de déterminer mois par mois la probabilité de dépassement d'un mou* vement de plate-forme d'amplitude donnée. L'analyse du fonctionnement du système à permis de fixer à $3 \mathrm{~m}$ l'amplitude maximale (crête à creux) du pilonnement en fonctionnement.

Au stade actuel des études et compte tenu de la précision des données, nous utilisons les hypothèses simplificatrices suivantes:

1) la barge filtre complètement les houles courtes (période inférieure ou égale à 6 secondes soit une longueur d'onde de 56 mètres) ;

2) en pilonnement, la barge suit les vagues exception: nelles d'un spectre de houle incident ; 
3) l'ensemble des vagues d'un spectre suit une distribution de probabilité de Rayleigh.

Sous ces hypothèses, la probabilité de dépasser un mouvement de pilonnement d'amplitude donnée se déduit directement de l'histogramme des amplitudes cumulées des houles de période supérieure à 6 secondes. Ces probabilités varient mensuellement dans de fortes proportions, de $3 \%$ en janvier à $10 \%$ en octobre avec une moyenne voisine de $5 \%$. Il convient de majorer ce temps de fonctionnement impossible des temps d'arrêt et mise en route et des temps d'entretien annuels. Globalement, le temps d'indisponibilité est de $10 \%$ du temps total.

Des solutions en plate-forme semi submersible auraient été beaucoup plus favorables, mais dans la gamme de puissances étudiées, leur bilan économique est fortement défavorable.

\section{Hydraulique interne}

La détermination du régime permanent des différents circuits internes par mer calme ne pose évidemment aucun problème mais il convient de s'assurer du comportement de ces circuits en régime transitoire lorsque la mer est agitée. Pour ce faire, on a élaboré des modèles mathématiques de simulation en adoptant un certain nombre d'hypothèses simplificatrices de manière à obtenir une précision suffisante à ce stade, sans avoir à faire appel à des méthodes très sophistiquées.
Ainsi, on a admis les approximations suivantes : - la houle est monochromatique ;

- les accélérations horizontales de la barge en mouvement sont faibles;

- la pression aux prises d'eau et au rejet n'est pas influencée par la structure et elle est donnée par la formule de la houle pure en profondeur infinie ;

- les pressions restent invariables dans les chambres d'évaporation et de condensation;

- l'inertie des pompes est négligée ;

- la densité de l'eau de mer est constante.

On a calculé les régimes transitoires consécutifs à l'apparition d'une houle succédant au calme plat. On a effectué un nombre suffisant de cycles de calcul (de 2 à 30) jusqu'à obtenir un régime périodique pratiquement établi.

Selon les circuits, on a utilisé les méthodes numériques d'intégration de Runge-Kutta ou de Newton.

Les résultats obtenus indiquent que le dispositif flotteur + flexible est apte à régulariser très efficacement l'apport d'eau froide.

Les débits d'eau chaude sont plus fluctuants, dans une gamme encore acceptable, sous réserve de vérifications supplémentaires, au voisinage de l'amplitude de houle maximale considérée, soit 3 mètres.

Ce régime pulsatoire fait apparaître un déséquilibre entre les circuits d'eau chaude et d'eau froide qui semble devoir aisément être corrigé par introduction d'une vanne réglable.

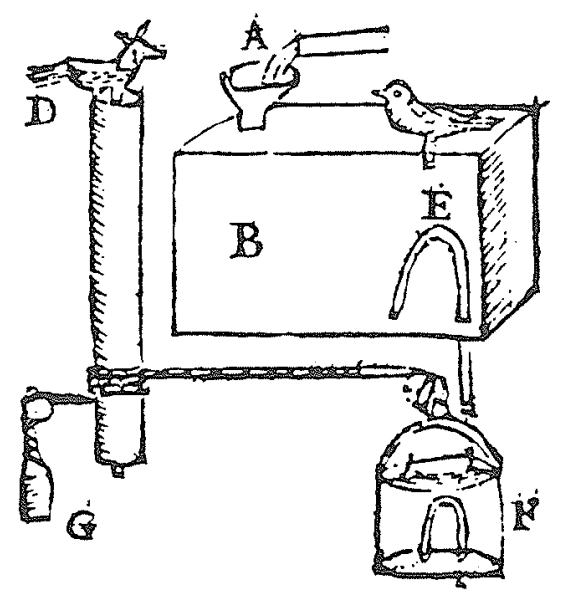

